\title{
Being in a Seclusion Room: The Forensic Psychiatric Inpatients' Perspective
}

Word count: 5000

\section{Louise Askew BSc Hons}

Trainee Clinical Psychologist

University of East Anglia

\section{Dr Paul Fisher}

Clinical Lecturer in Clinical Psychology and Specialist Clinical Psychologist

University of East Anglia

\section{Dr Peter Beazley}

Senior Clinical Tutor in Clinical Psychology

University of East Anglia

Ethical approval was obtained from the university's Faculty of Medicine and Health Sciences

Research Ethics Committee, the NHS Health Research Authority and the Research and

Development Committee of the NHS trust participants were recruited from.

This article has been accepted for publication and undergone full peer review but has not been through the copyediting, typesetting, pagination and proofreading process, which may lead to differences between this version and the Version of Record. Please cite this article as doi: 10.1111/JPM.12576

This article is protected by copyright. All rights reserved 
MRS. LOUISE ASKEW (Orcid ID : 0000-0003-4626-8865)

Article type : Original Article

\section{Corresponding author mail id: kmx15zeu@uea.ac.uk}

\section{Accessible Summary}

What is known on the subject

- Existing qualitative research has found inpatient service users to experience seclusion as highly distressing, with feelings of vulnerability, abuse and neglect often featuring in participants' accounts.

- The physical environment of the seclusion room and the interaction with clinical staff shape service users' personal seclusion experience.

What the paper adds to existing knowledge

- The majority of research on this topic focuses on seclusion within other restrictive practices. This paper provides new knowledge on one specific component of seclusion, the experience of being in the room, and draws attention to the specific psychological needs of service users during that aspect of their experience.

- This research provides new knowledge by exclusively exploring forensic inpatients' experience of the seclusion room, an under researched and often stigmatised population.

What are the implications to practice

- The findings support the need for a caring and non-threatening therapeutic interaction with a secluded service user for the duration of time they are in the seclusion room.

- The findings suggest that necessary nursing procedures, such as observations, should be carried out discretely and sensitively to avoid service users feeling abused and frightened. 


\begin{abstract}
Introduction: Contemporary qualitative research has explored service users' experience of seclusion and have found it to be a highly distressing and potentially traumatising experience for service users. The majority of the existing literature has researched seclusion within the context of other restrictive practices, resulting in findings that can only be considered an overview of the experience. The studies also rarely access participants with histories of considerable violence and imprisonment.
\end{abstract}

Question: What are forensic psychiatric inpatients' experience of being in a seclusion room?

Method: Seven inpatients in a medium secure hospital were interviewed and Interpretative Phenomenological Analysis (IPA) was used to analyse the data.

Results: Four superordinate themes were identified; 'intense fear', 'not getting the care I needed', 'I am being abused' and 'power struggle'.

Discussion: While participants were in the seclusion room they experienced extreme fear. Staff interaction played a considerable role in shaping the participants' experience. Staff actions were interpreted as neglectful and abusive. Participants experienced struggling for power with staff, seeking out power when left in a powerless position.

Implications for practice: These findings suggested that a carefully tailored therapeutic interaction is required during seclusion in order to safeguard the mental health of forensic inpatients.

Key words: Forensic, Patient Experience, Qualitative Methodology, Seclusion and Restraint.

\title{
Relevance Statement
}

The study reveals a novel in-depth understanding of one specific aspect of seclusion; being in the seclusion room. It offers a new insight into the experience of a complex client group (forensic inpatients) whose voices are often missing from the literature. The findings offer recommendations on how enhance clinical supervision and inform training for staff working in an emotionally challenging and hostile setting. The findings suggest the use of a psychological model to inform clinical supervision. Given the high level of responsibility 
nurses have with seclusion decision making and practice, the findings are likely to be of great interest.

\section{Introduction}

The modern day use of Restrictive Practices (RPs) to manage dangerous behaviour in psychiatric inpatient settings has been centre of attention in research, policies and television documentaries. Seclusion has been one of the RPs which has been the focus of several studies attempting to understand service users' experience of it in order to help inform the way it is implemented. Both clinicians and service users have expressed their concerns that seclusion could be re-traumatising already mentally unwell service users (Brophy et al., 2016 and Muir-Cochrane, O’Kane \& Oster, 2018), further heightening the value of such research.

Recent qualitative studies have aimed to gain an in-depth understanding of the service user perspective of seclusion, with findings suggesting an overwhelmingly negative experience. Studies have found the experience to be severely distressing for participants, with emotions such as fear, anger and humiliation emerging from the data (Ezeobelle et al., 2014; Faschingbauer et al., 2013, Haw et al., 2011; Kontio et al., Ling et al., 2015; Martinez et al., 1999; Natsaba \& Havenga, 2007 and Sambrano \& Cox, 2013). Findings suggest that participants experience the physical environment of the room as distressing, for example, studies by Ezeobelle et al., (2014), Haw et al., (2011) and Ntsaba and Havenga (2007) found participants experience the room negatively and similar to a prison cell. Participants' experience also appears to have been influenced by the interaction they received from staff. Faschingbauer et al., (2013) found that participants' experience improved when staff were attentive and explained what was happening. In contrast, Ezeobelle et al., (2014) found participants felt hopeless and powerless in response to a perceived lack of compassion from staff.

These studies reveal that seclusion has the potential to be highly traumatic for those who experience it, and that there are several complex components that contribute to the overall experience. While this body of research gives an insight into the service user experience, it is limited because in many studies, seclusion is considered alongside several different RPs (e.g. forced medication) in the same study (Brophy et al., 2016; Haw et al., 2011; Kontio et al., 2012; Larsen \& Terkelsen, 2014; Ling et al., 2015 and Mayers et al., 2010). The limitation 
this poses is that subtle and discrete aspects of seclusion, which may still be highly relevant to the service user experience, cannot be analysed in-depth. In addition, the majority of studies have large sample sizes (Brophy et al., 2016; Ezeobele et al., 2014; Haw et al., 2011; Kontio et al., 2012; Ling et al., 2015 and Mayers et al., 2010) restricting the depth of analysis, as indicated in Braun and Clarke (2013).

Faschingbauer et al., (2013), Natsaba and Havenga (2007) and Sambrano and Cox (2013) are three qualitative studies focusing specifically on psychiatric inpatients' experience of seclusion. Faschingbauer et al. (2013) had similar findings to what has been found in the other qualitative research exploring RPs. While the findings suggest that there are some similarities between seclusion experiences in various settings and with differing populations, there are limitations to this study that may have hindered its ability to collect a rich amount of data. The researchers maintained a specific and restricted inclusion criteria for their participants. Participants were required to be engaging in treatment and behaving prosocially on the ward. This is likely to have reduced the number of participants with persistent anti-social behaviour in the study's sample. This is relevant given the link between antisocial personality and aggressive behaviour, which is by definition more likely to lead to seclusion. Certainly, Vitacco et al.'s (2009) research found anti-social tendencies to be associated with aggressive behaviour. In addition, their research highlighted the complicated relationship between clinical presentation and type of aggression. For example, anger and active symptoms of mental illness predicted reactive aggression (e.g. responding to hallucinations) whereas psychopathic traits predicted planned and calculated aggression. Given that research has identified staff-patient relational factors to play a role in the seclusion experience (Ezeobelle et al., 2014; Faschingbauer et al., 2013; Ling, Cleverley \& Perivolaris, 2015 and Ntsaba \& Havenga, 2007), choosing a sampling methodology which underrepresented service users with anti-social characteristics may have resulted in a unique experience being absent from the findings.

Natsaba and Havenga's (2007) research used less restrictive inclusion criteria. Their findings were similar to Faschingbauer et al.'s (2013). However, additional themes regarding punishment and imprisonment emerged from the interviews. Their participants described feeling punished, and that the physical nature of the seclusion room triggered memories of being in a prison cell for those who had previously experienced imprisonment. While this research identified emotive themes, the in-depth personal meaning of seclusion was not 
explored. For example, while punishment was identified as a theme, the meaning and experience of what it was like to have felt punished, was not explored. The relationship between researcher and participant and how that influences recruitment, data collection or analysis was not reflected upon in their article. There may have been elements about this relationship that impacted on the findings which is missing from the analysis.

Sambrano and Cox (2013) conducted a phenomenological study into the service user experience of seclusion. Their small sample (three) enabled an in-depth reflection and consideration of the deeply personal meaning of the participants' experiences. Their research specifically explored the perspective of a marginalised group within society. They also identified themes surrounding abuse and neglect as well as complex power dynamics that existed between staff and service users during seclusion. They conclude their research by discussing how the societal context of the seclusion practice plays a role in service users' lived experience. It is recognised that both the external environment and relational factors play a role in the subjective seclusion experience. Sambrano and Cox's (2013) research has highlighted how seclusion experiences can be influenced by wider factors, such as the societal context.

While all three studies offer a more detailed exploration of the seclusion experience, they do not distinguish between the different aspects of seclusion. The lead up to seclusion, being in the room and the debrief after seclusion are incorporated together. The problem this poses is that there are different procedures occurring at these points and therefore, each component may have a different meaning and experience for participants. For example, the lead up to seclusion involves staff restraint by multiple staff members, being in the room involves isolation and the debrief is a one-to-one interaction.

The majority of research into seclusion is conducted with a narrow and specific sample of psychiatric inpatient service users from an acute hospital ward. One study (Haw et al., 2011) conducted research on a forensic psychiatric ward. The findings suggested that participants experience difficult thoughts and emotions during seclusion, that they find the environment unpleasant and worse than prison, but also that it can also be a positive opportunity for quiet reflection. 
By carrying out research in the forensic setting, Haw et al. (2011) had access to participants with different clinical needs and histories. The following factors may influence participants' experience of seclusion. Forensic inpatients will have had experience of prison, may have been incarcerated for several years, have committed serious interpersonal crimes and often be a group ostracised from society. Participants may have more anti-social interpersonal styles and be expected to engage in long term rehabilitation. Völlm et al. (2018) highlight the high prevalence of personality disorder (particularly anti-social and borderline) in long stay forensic inpatient settings (approximately 47\%). Service users with these personality disorders are often found to have more difficulty responding to rehabilitation (Bahorik \& Eack, 2010; McCarthy \& Duggan, 2010 and Stinson, 2016) than those with exclusively axis I disorders. While Haw et al.'s (2011) study potentially accesses new data, its qualitative methodology is hindered by the use of a large sample and data being only small extracts of interviews.

While it is recognised that seclusion is a necessary last resort in order to ensure staff and service user safety, on-going research is essential in order to positively influence the implementation. More in-depth understanding of the deeply personal meaning and experience of being in seclusion from the perspective of various client groups is required. In Brophey et al.'s (2016) research, participants expressed concern that marginalised and stigmatised groups would be more likely to receive RPs and would be more vulnerable to traumatisation from the experience.

Maguire, Young and Martin (2012) considered a stigmatised group in their study of a RP reduction programme. They evaluated the efficacy of a mainstream RP reduction programme applied to forensic hospitals. They found difficulties reducing seclusion in these hospitals and hypothesised that the personality traits of forensic inpatient service users are not amenable to the short term therapeutic interventions of mainstream RP reduction programmes. Currently, ostracised populations, such as forensic inpatient service users, are underrepresented in the research. Given that the behaviour of these service users may be one which is more likely to result in seclusion, and that this population may find responding to $\mathrm{RP}$ reduction programmes more difficult, it is a group that requires representation in the literature. Current practice may not accurately reflect the needs of these service users. 
In summary, the majority of contemporary research has given an overview of the seclusion experience, but within the context of various different aspects of RPs. Studies have also tended to exclude more complex presentations. This has resulted in a gap in the research. By researching a specific component of seclusion, it gives the opportunity for new features of the experience to be identified, leading to improved understanding of the experience for clinical recommendations as well highlighting areas for further research. This research focuses on one specific aspect of seclusion with an under researched population. It aims to answer the question, what are forensic inpatients' experience of being in a seclusion room?

\section{Method}

\section{Design}

Interpretative Phenomenological Analysis (IPA) focuses on how participants make sense of a personal lived experience, their reflections and the meaning they attach to it (Smith, Flowers and Larkin, 2012). This method enables in-depth understanding of the phenomenon from a deeply personal perspective. IPA is informed by hermeneutics, the theory of interpretation (Smith et al., 2012). It has a two stage interpretation process, with the researcher's role being to attempt to make sense of the participant trying to make sense of their experience, known as a double hermeneutic (Shaw, 2010). Epistemologically, IPA adopts a contextualist position, recognising that while the data analysis and results are grounded in the participants' own accounts, they are inevitably influenced by the researcher's own perceptions. All experiences have personal meaning and the interpretation of it will differ across time and context. As this study aimed to understand the individual personal experience of seclusion, IPA was deemed to be the best methodological approach to explore this phenomenon.

\section{Ethics}

Ethical approval was obtained from $* * *$

\section{Participants}


A small homogenous sample $(n=7)$, was recruited from a forensic medium secure hospital in England by one of two methods. The researcher was not a staff member and spent six days as a visitor on the wards, discussing the study and her role. They were asked by clinicians at the hospital if they would be interested in taking part and three consented to be contacted by the researcher. Two participants were recruited and one declined. The other five participants independently approached the researcher on the ward. Participants needed to have experienced seclusion in hospital as an adult, have mental capacity to consent and be orientated to time, place and person. In order to ensure participants were not put at risk of being retraumatised, only those whose most recent seclusion was 28 days or more prior to the interview were eligible. All participants were men of working age. They had all experienced seclusion more than once and they all had spent time in prison. Six participants were recruited from rehabilitation wards and one was recruited from an assessment ward. Participants were offered $£ 10$ for their contribution of time. This was in line with the hospital's protocol of reimbursing their service users for involvement in service development projects and the BPS Code of Human Research Ethics (2014) recommendation of a reasonable amount.

\section{Data collection}

The study was conducted over three wards. Data was collected through one-to-one semi-structured interviews with the lead author. The interview topic guide was developed in consultation with service users. It included questions about the description, the memory and the experience of being secluded in a seclusion room. All interviews took place on the ward where the participant resided in a quiet room. Interviews lasted between 25 and 105 minutes. They were recorded and then transcribed.

\section{Analysis and quality assurance}

Analysis followed Smith et al.'s (2012) guidelines and was carried out in a systematic format, moving back and forth through different ways of thinking about the data, which was documented to provide an audit trail. The first stage involved the researcher reading and immersing into the data and noting anything of importance in a descriptive and interpretative sense. The second stage involved developing initial themes and then searching the transcript for connections between themes. This process was carried out for each transcript 
individually and then the researcher looked for patterns and connections across all the transcripts.

Yardley (2000) offers flexible principles of good quality research and these were applied to the research process and write. The principles were interpreted and applied with the contextualist position. The researcher regularly reflected on the process during the interview stage and throughout the analysis to ensure that the analysis was grounded in participants' data. This was facilitated by the use of a reflective journal and discussions with supervisors. The quality was further enriched through triangulation by the researcher meeting with another IPA researcher to review coding and theme development, as per Yardley's (2015) recommendations. All aspects of the reflection and supervision were documented in order to ensure the auditability of analysis.

\section{Reflexivity}

Due to the complex nature of undertaking emotive research in a secure and potentially hostile setting, it is essential to reflect on dynamics and interpersonal factors that may have contributed to the analysis. The presence of a power imbalance throughout data collection was unavoidable, and was commented on by one participant when he highlighted his awareness of not having keys when the researcher did. During the interviews, the power struggle was often played out in the dynamics between researcher and participant. For example, the researcher asking questions and recording the interview gave the researcher power, the participant raising his voice, altering his body posture or choosing to talk off topic, gave the participant power. These observations have benefitted the research, as they have been used to enrich the interpretive nature of the analysis.

\section{Findings}

The analysis revealed four superordinate themes; intense fear, not getting the care I needed, I am being abused and power struggle.

\section{Intense fear}


Whilst in the seclusion room, participants had an experience of intense fear. This fear was related to the physical aspects of the seclusion experience, which included staff and the environment. It was also associated with participants' own thoughts. Participants described an experience of ruminating whilst in the room and felt fearful of these thoughts.

Ali talked about how staff needed to enter the room while he was secluded because he covered the observation panel and hid. The extremeness of his fear was apparent during interview when he talked about his disturbing thoughts that he may be killed.

...every time they open the door, they kinda like all in gloves and there was about 12 of them, I thought, what the fuck's going on here, that's why I was getting you know like ideas in my head thinking they're gonna fuckin' kill me.

Liam, who was the only participant to disclose a traumatic experience of childhood abuse, described how he felt anxious while in seclusion and that this was related to him being engrossed in his own thoughts. His thoughts initially revolved around the potential immediate consequences of his behaviour prior to seclusion and then progressed to thoughts about long term consequences and future behaviour.

Worrying that, I might do it again, d'ya know what I mean? Or praps, worse... Yeah... Well like, hurting someone worse, and get in even more trouble.

\section{Not getting the care I needed}

All participants experienced a feeling of neglect. Participants directly commented on this or described needing some form of basic care (e.g. warmth) that they did not get.

Feeling neglected by both the room and staff was highly central to Aaron's experience. He spoke about a feeling of abandonment. He described being left, (by staff) without basic needs being addressed and that this was a horrible experience. The use of the word left and by describing it this way demonstrates that this was a feeling of neglect, rather than using an un-emotive statement to acknowledge that there was only him in the room.

Left in a seclusion room for a week without my clothes. I shit up the walls. 
Jay, who of all participants, had spent the least about of time in hospital, more explicitly described feeling alone and abandoned, which challenged his expectation of care in a hospital setting.

I just remember being really distressed... Makes you more, made me more, determined that, I'm really on my own. And, seems no matter where you get put for care, ultimately, there is no help. It's just feels totally like, abandoned, helpless...

\section{I am being abused}

Participants reflected on how the actions of staff were sometimes interpreted as a form of abuse. Participants often discussed these experiences alongside themes of fear. Abuse experiences ranged from physical abuse to abuse of a sexual nature. No participants made allegations of actual abusive behaviour. However, they described interpreting the behaviour of staff when they entered the room or the fact that staff constantly observed them, as feeling as though they were a victim of staff's abuse. Staff entering the room to access things such as a bed pan or to do an assessment was experienced in some cases as a form of abuse. For example, as a result of Peri's unsafe behaviour while in the seclusion room, some of the furnishings had to be removed. While he felt that this was a necessary action of staff, he explained that it left him feeling abused.

Two participants described feeling violated sexually as a result of being observed using the bathroom. Ali described having a vivid visual memory of staff observing him in the shower. He experienced this as a form of abuse.

Ifelt like I was being like, visually abuse or something. It didn't feel, feeling right at all.

For some participants, staff's behaviour was interpreted as a deliberate act to neglect their basic needs. For example, Avie's described the room as cold and that he was not given a blanket. While feeling desperate for care, he interpreted this as a cruel and deliberate act of neglect. This is demonstrated by his use of the word abusing, with abuse being associated with cruelty and deliberate action on the part of a perpetrator. Rather than commenting on 
being without a blanket, he commented on staff not providing him with one, implying that he felt staff had decided, on purpose, to neglect him.

$$
\text { Ifelt that they're, that's some sort of abusing me. }
$$

\section{Power struggle}

Participants experienced a loss and gain of power throughout seclusion. When they experienced powerlessness, they were in a vulnerable position and staff were perceived to be in a powerful position. Participants felt that staff had control over their seclusion experience and the duration of it. This led to participants behaving passively in the hope that it would result in them getting what they wanted e.g. to leave the room. Both David and Avie described actively trying not to argue with staff for fear of the consequences, e.g. staying in seclusion for longer or being restrained.

This theme was highly central to Peri's experience. Of all the participants, he had spent the longest amount of time incarcerated (in high secure prison and high and medium secure hospital) which was almost half of his life. He reflected on his experience of power in those institutions. He then considered several seclusion experiences and felt that he deliberately sought out power as a result of feeling powerless. For example, by refusing to leave the seclusion room and openly masturbating. He explained why he felt the need to do that.

In a place where all the control is taken off you, yeah, you've suddenly got a bit of control. 'Cause I think that's the thing, if you're in an environment that is controlled on every level, yeah, I think, in a way, it creates behaviours in people, yeah, 'cause they need someeveryone needs a level, some people are comp, complete control freaks, yeah, and need every aspect of their life controlled, to have control of it, yeah? But then, then you're in an environment where you don't have any control, everybody's trying to grab that little bit of control.

Of all the participants, the presence of a power dynamic was most strongly felt during David's interview. He less explicitly described his struggle to gain power but shared reflections which were interpreted to be so. For example, as a way to feel powerful, he 
assessed the capabilities, qualifications and salaries of staff in response to staff assessing his risk and felt that a lack of training explained the long duration of his seclusion.

a nurse is not gonna take the risk, of saying right well I think he's fine...A doctor they have a lot more training they can, sit there and look at it psychiatrically, if need be, given them medication. Nurses can't do that.

\section{Discussion}

The aim of this research was to unearth the deeply personal experience of being in a seclusion room as a forensic psychiatric inpatient. The analysis revealed four super ordinate themes that capture the participants' key experiences whilst in the room.

Being in the seclusion room was a frightening experience, independent of duration or frequency. Some participants felt victims of staff's abuse, which represents immense powerlessness. Participants desired care, with some openly disclosing this and others describing an experience of unmet needs. Some of their emotive language demonstrated that they wanted their needs to be addressed (be that physical or emotional) but instead they felt they received neglect and abandonment. Participants desperately sought out ways to feel powerful in order to have a sense of control. Some of this was through disruptive behaviour.

Previous research has found fear to be a prevalent, overwhelming emotion for nonforensic inpatients while in a seclusion room (Kontio et al., 2012; Martinez et al., 1999; Ntsaba \& Havenga, 2007 and Steinert et al, 2013). Ntsaba and Havenga (2007) discuss how elements of the environment led to secluded service users being so fearful that they felt their life was in danger. However, they also identify how their participants' fear stemmed from anticipation of what might happen next. Ling et al (2015) and Wynn (2004) explore participants' experience of restraint and their findings also identify fear stemming from participants' thoughts about what could happen (e.g. being restrained again while asleep). The present study suggests that fear is prevalent for a forensic inpatient population also, and that it is highly intense whilst in the room. These findings, alongside the above mentioned research, propose that fear during seclusion cannot be explained by environmental factors alone and that there are powerful personal internal strategies (e.g. rumination) initiating or 
maintaining fear. Given the mental state of service users during seclusion, the intensity of these internal factors are likely to be high.

Experiencing a lack of care is a theme that has arisen in several studies exploring seclusion experiences (Faschingerbauer et al., 2013; Haw et al., 2011; Ling et al, 2015 and Ntsaba \& Havenga, 2007) and has also been highlighted by nursing staff as a concern they have about how seclusion is implemented (Alty, 1997). In the present study, being without care left participants feeling neglected and abandoned, something Ezeobelle et al.'s (2014) participants also reported. These commonalities amongst studies span over several years, countries and hospital environments, suggesting that recent changes to seclusion practice may not be addressing the factors that influence service users' experience of care. It may also be that there are common characteristics of service users who experience seclusion that makes them more vulnerable to feeling neglected, such as histories of abuse (Hammer et al., 2011), psychotic symptomology (Keski-Valkama et al., 2010) or being a marginalised group within society (Happell \& Koehn, 2010). For the participants in the present study, the experience of care centred around their experience of staff.

Feeling as though the seclusion experience is abusive has been identified in earlier research. For some participants of that research, they reported incidents of emotional and physical abuse from staff (Ntsaba \& Havenga, 2007). In the present study, no accusations were made regarding abuse, but participants described experiencing staff as though they were perpetrating abuse against them. This highlights that actual abuse does not need to occur in order for a service user to feel as though they have experienced the trauma of abuse. This experience left participants in a vulnerable position, experiencing intense fear and feeling neglected. The participants' interviews that contributed to the, 'I am being abused theme' included their stories of seclusion that occurred both prior to the introduction of the 2015 NICE guidelines and after. This suggests that this is an experience that transcends various policy attempts to ensure ethical seclusion use, and is more related to the deeply personal meaning.

Previous literature exploring various coercive inpatient experiences has revealed themes centring around the powerlessness of service users (Haw et al., 2000; Meehan, Vermeer \& Windsor, 2000; Ryan \& Happell, 2009 and Sibitz et al., 2011). These studies have described participants' experience of a loss of control and power imbalance between 
staff and service users. The current study revealed a power dynamic, described as a power struggle, that has been less identified in previous research into the experience of RIs. A minimal amount studies have found suggestions of a power struggle dynamic. Meehan et al. (2000) found that service users were able to cope with the loss of control by engaging in behaviour that did not necessarily reflect how they were feeling, but was a way to "play the game" and be released from seclusion. This was referring to passive behaviour where service users presented themselves as powerless, but ultimately were misleading staff which subtly shifts the power in the direction of the service user. Some of the more overt power-seeking behaviours, like those identified in the current study, have been captured in previous seclusion research, for example, masturbation in seclusion (Kehl Richardson, 1987) and refusing to eat (Sambrano \& Cox, 2013). In Sambrano and Cox's (2013) study, the procedure of seclusion was somewhat different to in other research, as police transferred them into the seclusion room rather than clinicians. Two of the three participants had had experiences of prison prior to hospital. These are forensic elements that may not be present in other studies.

The nature of a forensic population may be a key influencing factor to the power struggle that is apparent in the findings of this study. Within forensic psychiatry, Holmes (2002) used grounded theory to explore the power dynamics between nurses and service users. He found that overall, nurses constantly hold a powerful position over service users in the secure environment. The participants (nurses) felt that due to having a role as disciplinarian and carer, it made it impossible to have a therapeutic relationship of equal power. While they felt that they were able to give service users some freedom or control, this was carefully calculated and ultimately had the intention of ensuring nursing staff could have the ability to control their service users' behaviour in the long term. Holmes identified that service users may develop ways to gain power over nurses, for example, by remaining silent during therapeutic meetings. While Haw et al.'s (2011) study in a forensic hospital did not identify themes alluding to service users gaining power, this may be a reflection of the methodological approach (large sample size and minimal quantities of qualitative data) which limited the extent to which participants' accounts could be analysed.

This research has highlighted the deeply personal experience of seclusion and that there may be factors related to service users' mental health and history that contribute to their experience. It has drawn attention to how personal experiences are not always openly shared 
by service users, meaning that presumptions should not be made regarding a service user's experience. The Power Threat Meaning Framework (PTM) (Johnstone and Boyle, 2018) can be used to understand the interaction between the themes. According to PTM, participants may experience seclusion as a trauma that is interpreted as threatening. They cope with the threat using long standing survival strategies (e.g. seeking power).

\section{Limitations}

It is recognised that the researcher not being a staff member may have meant that participants felt more able to open up honestly, it could alternatively have meant that participants were not able to build enough rapport to elaborate as much as they would have done with a familiar staff member. This means that it possible that there was some data that the researcher was unable to access.

It is also possible that participants' recollections of their experiences are inaccurate. Some participants spoke about seclusion experiences from several years ago. Smith and Gudjonsson's (1995) study with forensic inpatients found that the presence of anxiety hinders patients' ability to remember information, which in turn increases the chance of confabulation. The findings of their study are relevant, given that fear was a core part of participants' experience. However, this was not considered to be a considerable limitation as analysis focuses on the meaning, reflections and interpretation participants place on their experience. Therefore, potentially inaccurate memories are part of the experience, as it is the memories (inaccurate or accurate) that is what is influencing the meaning participants place on it.

\section{Implications for Practice}

This study illuminates the emotional experience and interpersonal dynamics that are present for participants whilst secluded. The complex interaction between the themes should be considered when seclusion is implemented and how service users' behaviour can be affected by aspects that may have previously gone unnoticed by staff. This highlights the need to ensure that therapeutic interaction is prioritised during the seclusion process, carefully tailoring this interaction to meet the needs (which may be unspoken) of the service user. 
The use of necessary nursing procedures (e.g. observation or assessment) should be applied sensitively, taking into consideration the history of the service user and their mental health difficulties. Staff training on how to formulate service users' behaviour using a theoretical model such as PTM, can help staff individually tailor their therapeutic response to such behaviour.

The importance of the power struggle that this study highlights, gives further insight into how to improve nursing practice. A staff response which focuses on regaining more power is likely to result in increased in power-seeking behaviour from service users. Rather, staff should provide care, considering that service users may feel frightened, neglected and abused. In order to help staff develop therapeutic skills, ensuring clinical supervision allows space for staff to reflect on their experience of secluding a service user may help them to identify any power seeking behaviour they unintentionally engage in. 


\section{References}

Bahorik, A. L., \& Eack, S. M. (2010). Examining the course and outcome of individuals diagnosed with schizophrenia and comorbid borderline personality disorder. Schizophrenia Research, 124(1), 29-35. https://doi.org/10.1016/j.schres.2010.09.005

Braun, V., \& Clark, V. (2013). Successful qualitative research: A practical guide for beginners. London: Sage Publications Ltd.

British Psychological Society. (2014). Code of Human Research Ethics.

Brophy, L. M., Roper, C. E., Hamilton, B. E., Tellez, J. J., \& McSherry, B. M. (2016). Consumers and Carer perspectives on poor practice and the use of seclusion and restraint in mental health settings: Results from Australian focus groups. International Journal of Mental Health Systems, 10(6), 1-10.

Ezeobele, I. E., Malecha, A. T., Mock, A., Mackey-Godine, A., \& Hughes, M. (2014). Patients' lived seclusion experience in acute psychiatric hospital in the United States: A qualitative study. Journal of Psychiatric and Mental Health Nursing, 21(4), 303-312. https://doi.org/10.1111/jpm.12097

Faschingbauer, K., Peden-McAlpine, C \& Temple, W. (2013). Use of seclusion: Finding the voice of the patient to influence practice. Journal of Psychosocial Nursing, 51(7), 3238.

Hammer, J. H., Beck, N. C., Menditto, A., \& Coleman, J. (2011). The Relationship Between Seclusion and Restraint Use and Childhood Abuse Among Psychiatric Inpatients. Journal of Interpersonal Violence, 26(3), 567 - 579. https://doi.org/10.1177/0886260510363419

Happell, B., \& Koehn, S. (2010). From numbers to understanding: The impact of demographic factors on seclusion rates. International Journal of Mental Health Nursing, 19(3), 169-176. https://doi.org/10.1111/j.1447-0349.2010.00670.x

Haw, C., Stubbs, J., Bickle, A., \& Stewart, I. (2011). Coercive treatments in forensic psychiatry: A study of patients' experiences and preferences. Journal of Forensic 
Psychiatry and Psychology, 22(4), 564-585.

https://doi.org/10.1080/14789949.2011.602097

Holmes, D. (2002). Police and pastoral power : governmentality and correctional forensic psychiatric nursing. Nursing Inquiry, 9(2), 84-92.

Keski-Valkama, A., Sailas, E., Eronen, M., Koivisto, A.M., Lönnqvist, J. \& Kaltiala-Heino, R. (2010). Who are the restrained and secluded patients : a 15-year nationwide study. Social Psychiatry \& Psychiatric Epidemiology, 45(11), 1087-1093. https://doi.org/10.1007/s00127-009-0150-1

Kontio, R., Joffe, G., Putkonen, H., Kuosmanen, L., Hane, K., Holi, M., \& Välimäki, M. (2012). Seclusion and restraint in psychiatry: Patients' experiences and practical suggestions on how to improve practices and use alternatives. Perspectives in Psychiatric Care, 48(1), 16-24. https://doi.org/10.1111/j.1744-6163.2010.00301.x

Larsen, I. B., \& Terkelsen, T. B. (2014). Coercion in a locked psychiatric ward. Nursing Ethics, 21(4), 426-436. https://doi.org/10.1177/0969733013503601

Ling, S., Cleverley, K., \& Perivolaris, A. (2015). Understanding Mental Health Service User Experiences of Restraint through Debriefing: A Qualitative Analysis. The Canadian Journal of Psychiatry, 60(9), 386-392. https://doi.org/10.1177/070674371506000903

Maguire, T., Young, R., \& Martin, T. (2012). Seclusion reduction in a forensic mental health setting. Journal of Psychiatric and Mental Health Nursing, 19(2), 97-106. https://doi.org/10.1111/j.1365-2850.2011.01753.x

Martinez, R., Grimm, M., \& Adamson, M. (1999). From the other side of the door: patient views of seclusion. Journal of Psychosocial Nursing \& Mental Health Services, 37(3), $13-22$.

Mayers, P. A. T., Keet, N., Winkler, G., \& Flisher, A. J. (2010). Mental health service users' perceptions and experiences of sedation, seclusion and restraint. International Journal of Social Psychiatry, 56(1), 60-73. https://doi.org/10.1177/0020764008098293 
McCarthy, L., \& Duggan, C. (2010). Engagement in a medium secure personality disorder service: A comparative study of psychological functioning and offending outcomes. Criminal Behaviour and Mental Health, 20, 112-128. https://doi.org/10.1002/cbm

Muir-Cochrane, E., O’Kane, D., \& Oster, C. (2018). Fear and blame in mental health nurses' accounts of restrictive practices: Implications for the elimination of seclusion and restraint. International Journal of Mental Health Nursing. https://10.1111/inm.12451

Ntsaba, G. M., \& Havenga, Y. (2008). Psychiatric in-patients' experience of being secluded in a specific hospital in Lesotho. Health SA Gesondheid, 12(4), 3-12. https://doi.org/10.4102/hsag.v12i4.267

Sambrano, R., \& Cox, L. (2013). "I sang Amazing Grace for about 3 hours that day": Understanding Indigenous Australians' experience of seclusion. International Journal of Mental Health Nursing, 22(6), 522-531. https://doi.org/10.1111/inm.12015

Shaw, R. (2010). Interpretative Phenomenological Analysis. In M. Forrester (Ed.) Doing qualitative research in psychology: A practical guide. (pp. 177 - 201). London: Sage Publications Ltd.

Smith, J. A., Flowers, P., \& Larkin, M. (2012). Interpretative phenomenological analysis: Theory, method and research. London: Sage Publications Ltd.

Smith, P., \& Gudjonsson, G. H. (1995). Confabulation among forensic inpatients and its relationship with memory, suggestibility, compliance, anxiety, and self-esteem. Personality and Individual Differences, 19(4), 517-523. https://doi.org/10.1016/01918869(95)00085-K

Stinson, J. D. (2016). Predictors of Treatment Noncompletion in a Sample of Inpatient Sex Offenders With Serious Mental Illness. Psychiatric Services, 67(1), 43-48. https://doi.org/10.1176/appi.ps.201400415

The National Institute for Health and Care Excellence. (2015). Violence and aggression: short-term management in mental health, health and community settings (NG10). Retrieved from https://www.nice.org.uk/guidance/ng10/resources/violence-and- 
aggression-shortterm-management-in-mental-health-health-and-community-settings1837264712389

Vitacco, M. J., Van Rybroek, G. J., Rogstad, J. E., Yahr, L. E., Tomony, J. D., \& Saewert, E. (2009). Predicting short-term institutional aggression in forensic patients: A multi-trait method for understanding subtypes of aggression. Law and Human Behavior, 33(4), 308-319. https://doi.org/10.1007/s10979-008-9155-7

Völlm, B. A., Edworthy, R., Huband, N., Talbot, E., Majid, S., Holley, J., ... Duggan, C. (2018). Characteristics and pathways of long-stay patients in high and medium secure settings in England; A secondary publication from a large mixed-methods study. Frontiers in Psychiatry, 9, 1-12. https://doi.org/10.3389/fpsyt.2018.00140

Yardley, L. (2000). Dilemmas in qualitative research. Psychology and Health, 15(2), 215228.

Yardley, L. (2015). Demonstrating. In J. A. Smith (Ed.), Qualitative psychology: A practical guide to research methods (pp. 257-272). London: Sage Publications Ltd. 